Rev. Bras. Cir. Cardiovasc.,

1(2):29-33, 1986

\title{
Contrapulsação aórtica intraoperatória pós circulação extracorpórea (CEC): apresentação de método
}

Celso Luis dos REIS*, Paulo Roberto Barbosa ÉVORA*, Paulo José de Freitas RIBEIRO*, José Carlos Franco BRASIL*, Adonis Garcia OTAVIANO*, Hércules Lisboa BONGIOVANI*, Rubio BOMBONATO*, Almir Sales PEREIRA*

RBCCV

REIS, C. L.; ÉVORA, P. R. B.; RIBEIRO, P. J. F.; BRASIL, J. C. F.; OTAVIANO, A. G. ; BONGIOVANI, H. L.; BOMBONATO, R.; PEREIRA. A. S. - Contrapulsação aórtica intraoperatória pós circulaçăo extracorpórea (CEC): apresentaçāo de método. Rev. Bras. Cir. Cardiovas., 1(2):29-33, 1986.

RESUMO: Apresenta-se um sistema para obtenção do efeito de contrapulsação aórtica no período intraoperatório pós perfusional de cirurgias cardiacas.

DESCRITORES: contrapulsação aórtica, intraoperatória; contrapulsação aórtica; circulação extracorpórea.

\section{INTRODUÇÃO}

Corações submetidos a procedimentos cirúrgicos com circulação extracorpórea (CEC), freqüentemente apresentam comprometimento da função ventricular, ao reassumir seus batimentos próprios. Nestas ocasiōes, torna-se necessária a utilização de apoio farmacológico e/ou mecânico até que a função cardíaca se apresente adequada. $\mathrm{O}$ uso de cardiotônicos, imediatamente após a saída de CEC, deve ser evitado como uma das maneiras de prevenção dos danos associados à reperfusão miocárdica. Logo, a possibilidade do apoio circulatório mecânico deve ser considerada. A assistência circulatória mecânica tem sido, classicamente, realizada pela técnica de contrapulsação aórtica, utilizando-se o balāo intra-aórtico.

O presente trabalho tem por objetivo apresentar um sistema para obtenção do efeito de contrapulsação aórtica no período intra-operatório pós- perfusional. Em substituição ao catéter balão intra-aórtico, utiliza-se a própria cânula da perfusão aórtica ainda conectada à bomba de CEC, acoplada a um dispositivo gerador de fluxo pulsátil. Este sistema original para obtenção de pulsações em CEC tem sido de uso regular, desde 1981, durante cirurgias cardiacas que necessitam de perfusões prolongadas. Os seus resultados funcionais hemodinâmicos e metabólicos já foram objetos de comunicaçōes anteriores ${ }^{3-6}$.

\section{MATERIAL E MÉTODO}

O dispositivo para fluxo pulsátil (DFP) é interposto entre a saída da linha arterial de uma bomba de roletes comum e a cânula, inserida na raíz da aorta, cujo diâmetro da porção intra-arterial é de 0,8 $\mathrm{cm}$. O DFP consta de um bulbo de borracha atóxica 
REIS, C. L.; EVVORA, P. R. B.; RIBEIRO, P. J. F.; BRASIL, J. C. F.; OTAVIANO, A. G.; BONGIOVANI, H. L.; BOMBONATO. R.: PEREIRA, A. S. - Contrapulsaçăo aórtica intraoperatória pós circulação extracorpórea (CEC): apresentaçāo de método. Rev. Bras. Cir. Cardiovasc., 1(2):29-33, 1986.

contido dentro de um recipiente rígido, juntamente com um balāo de látex inflável, sendo o recipiente preenchido por água. O pulso é gerado pela compressão do bulbo de borracha por meio da insuflação do balão de látex, que é ligado a uma bomba pneumática acoplada à bomba de roletes. Esta bomba pneumática é construída com o auxilio de uma seringa de vidro de $50 \mathrm{ml}$, cujo êmbolo funciona como pistão, montado em um suporte metálico adequado e que permite um ajuste prévio do volume de ar. Este volume bombeado insuflará o balão de látex do DFP, o qual comprimirá o balâo de borracha por onde passa o sangue arterial, gerando a onda de pulso. O êmbolo da seringa mantém-se sempre sob traçăo por uma mola e é acionado pela manivela do rolete arterial, gerando, portanto, um pulso a cada volta do rolete. Ressalta-se que todo o sistema é totalmente desprovido de válvulas.

Como ampliação da utilização do método, procurou-se associar o auxílio mecânico à circulação aos benefícios obtidos pela CEC com fluxo pulsátil. Este auxilio cardiocirculatório foi conseguido obtendo-se o efeito de contrapulsação aórtica pela substituição da insuflação do balão de látex do DFP, que era feita pela seringa acoplada ao rolete arterial, por uma bomba eletromagnética sincronizada com o ECG do paciente *. Para que não haja amortecimentos, pinça-se o segmento da linha arterial

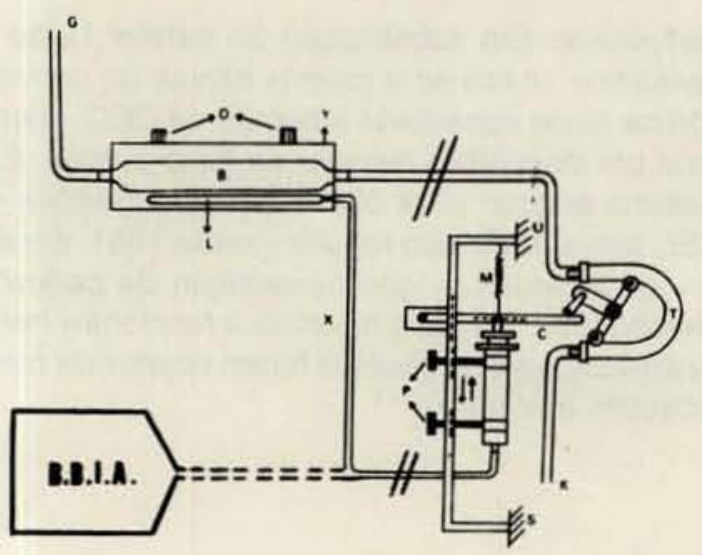

Fig. 1 - Representaçăo esquemática do sistema mecânico para produçăo do fluxo pulsátil em CEC adaptado para contrapulsaçāo aórtica intra-operatória: $\mathrm{V}$ e $\mathrm{S}$ - conexâo ao chassis da máquina de CEC: T - tubo de látex da linha arterial usual; $\mathrm{C}$ - alavanca que aciona o sistema de compressāo (seringa de $50 \mathrm{ml}$.): $\mathrm{M}$ - mola de retorno; $\mathrm{K}$ - conexảo ao oxigenador; $\mathrm{B}$ - bulbo elástico de borracha atóxica; $\mathrm{L}$ - balâo de látex; $\mathrm{X}$ - catéter de conexăo entre o sistema de compressão e o balăo de látex: Y - recipiente de plástico rígido; $\mathrm{O}$ - orificio para introduçăo de água no recipiente y; $\mathrm{G}$ - conexăo à cânula de perfusâo na aorta: B. B. I. A. - bomba de balấo intra-aónico. entre o DFP e a bomba de roletes (Figura 1). Todo o procedimento é assumido pelo próprio perfusionista, sem qualquer alteração no campo operatório. O rendimento hemodinâmico do efeito de contrapulsação será máximo fazendo-se com que o pico de pressão positiva da bomba coincida com o terço proximal ao nó dicrótico da distância entre este e o pico sistólico imediatamente anterior, ou seja, adotando-se o mesmo procedimento utilizado para o bombeamento com o balāo intra-aórtico (Figura 2). Tal ajuste é feito pela monitorização do perfil do pulso obtido da artéria radial, puncionada ou dissecada, e conectada, através de um transdutor de pressāo, a um cardioscópio de dois canais, dos quais se utilizou apenas o canal para registro das pressōes, de onde foram tomadas fotografias para documentação dos efeitos obtidos. Para comparações, apresentam-se os perfís de pulso obtidos na artéria radial em situação fisiológica (Figura 3), em CEC com fluxo pulsátil (Figura 4) e o perfil de pulso mecanicamente assistido pelo método proposto (Figura 5).

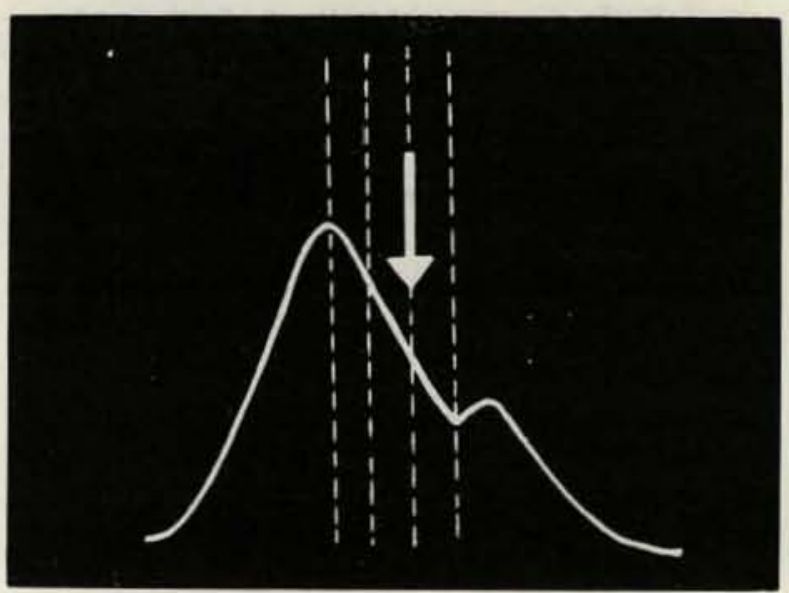

Fig. 2 - Diagrama esquemático de perfil năo assistido mecanicamente, na artéria radial. A seta indica o ponto adequado, na curva de pulso, com o qual deve coincidir o pico de pressão positiva da bomba.
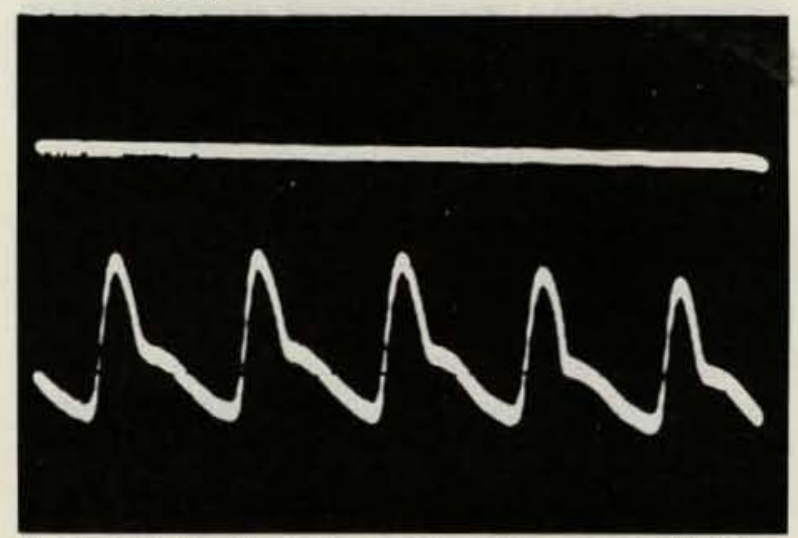

Fig. 3 - Perfil do pulso obtido na artéria radial em situaçăo fisiológica. 
REIS, C. L.; EVORA, P. R. B.; RIBEIRO, P. J. F.; BRASIL, J. C. F.; OTAVIANO, A. G.; BONGIOVANI, H. L.; BOMBONATO, R.; PEREIRA, A. S. - Contrapulsação aórtica intraoperatória pós circulação extracorpórea (CEC): apresentação de método. Rev. Bras. Cir. Cardiovasc, 1(2):29-33, 1986.

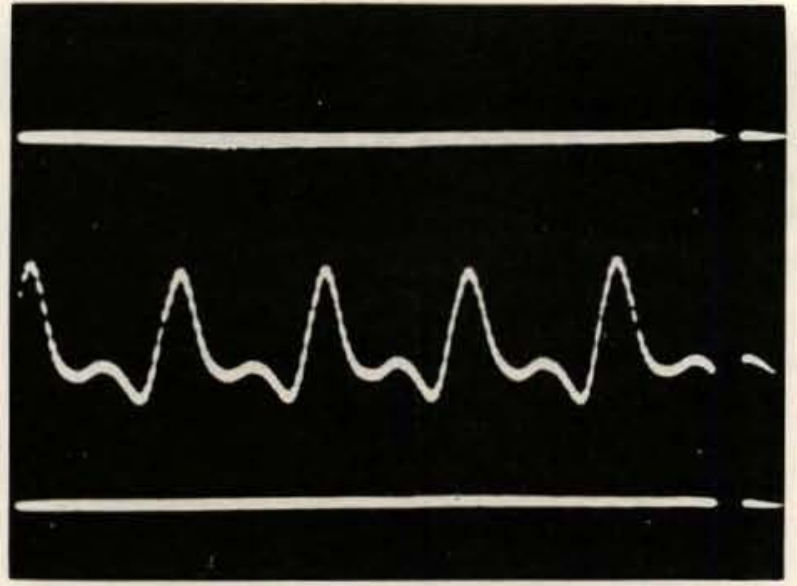

Fig. 4-Perfil do pulso obtido na antéria radial durante a CEC com fluxo pulsatil.

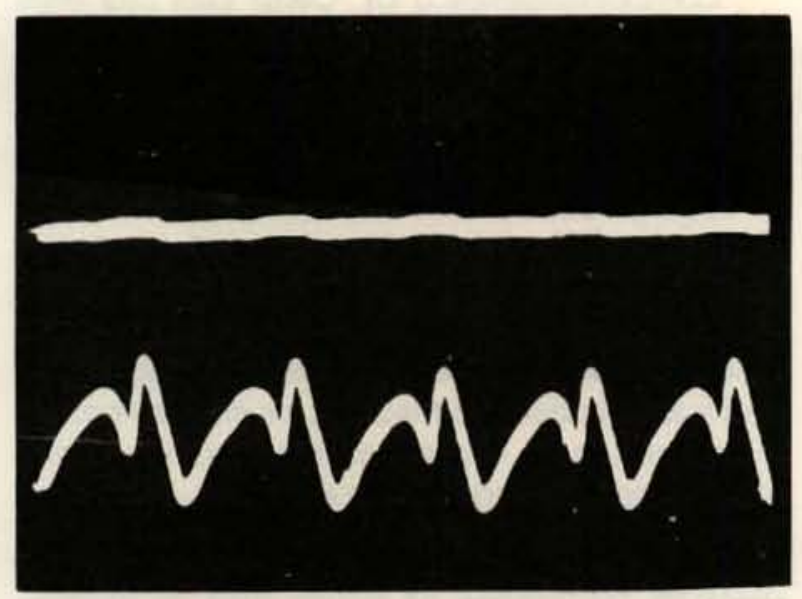

Fig. 5 - Perfil do pulso obtido na artéria radial mecanicamente assistido pela contrapulsação aórtica

\section{COMENTÁRIOS}

Os benefícios oferecidos pela assistência circulatória mecânica por contrapulsação com balāo intra-aórtico na síndrome de baixo débito cardiaco já sâo bem conhecidos. Estes benefícios podem ser de tal monta que chegam a modificar, significantemente, o prognóstico desta entidade clínica, principalmente quando desencadeada no período pós perfusional em cirurgia cardiaca ${ }^{2}$. Embora seja verdade que, na maioria das vezes, o bombeamento diastólico deva ser um procedimento demorado até por vários dias com auxilio do balão intra-aórtico, o seu uso por curto período, imediatamente após a CEC, pode ser útil?
O método de retirar um certo volume de sangue do compartimento arterial pela artéria femoral, durante a sístole, e impulsioná-lo de volta durante a diástole já foi descrito' ${ }^{\prime}$ Sua aplicação foi limitada pela lesão das células sangüineas e pela própria incapacidade do leito arterial próximo à cânula femoral de manter-se pérvio, durante a fase de pressão negativa aplicada pela bomba sincronizada. Desta maneira, pode ocorrer que o volume removido durante a sístole altere pouco a impedância a ser vencida pelo ventrículo esquerdo durante a ejeção sistólica. Sendo a cânula de perfusão pela aorta de grosso calibre e sendo a própria aorta um vaso de grande diametro, a lesão das células sangüíneas fica minimizada e não se observa colapso da artéria, o que torna o método apresentado perfeitamente viável.

Até o momento, este procedimento foi utilizado em 8 pacientes que não apresentavam contra-indicações para contrapulsação aórtica e que demonstraram grande instabilidade cardiocirculatória após a suspensão da CEC para revascularizaçāo do miocárdio. Observaram-se, em todos eles, hipocontratilidade cardíaca e incapacidade de manter pressão arterial média de $60 \mathrm{~mm} / \mathrm{Hg}$, ou mais, e niveis de pressão de átrio esquerdo inferiores a 20 $\mathrm{cmH}_{2} \mathrm{O}$. Quatro desses pacientes necessitaram, depois, de assistência circulatória mecânica prolongada e receberam a inserção do catéter balão intraaórtico. Nos outros 4 pacientes, a contrapulsação intra-operatória, obtida pelo método descrito, foi mantida por período que variou de 30 a 60 minutos, conseguindo-se uma recuperação da função cardiaca sem a necessidade posterior de qualquer apoio mecânico à circulação.

A finalidade maior do método foi a substituiçāo do auxílio mecânico, obtido pelo retorno à CEC, pela contrapulsação aórtica. No apoio circulatório com a CEC, o alívio da sobrecarga volêmica tem repercussão positiva, primariamente, na função do ventrículo direito e sobre a pressão arterial média que a bomba ajuda a manter. Com a contrapulsação aórtica, as variações de pressão ocorridas na aorta facilitam o esvaziamento do ventrículo esquerdo pela diminuição da pós carga, além de contribuir para melhorar a perfusão coronária pelo aumento da pressão diastólica aórtica. Além disso, o comprimento do circuito de CEC percorrido pelo sangue é muito menor, ficando abolida a sua propulsāo por roletes. Trata-se, portanto, de um importante recurso para a descontinuaçāo da CEC, com diminuição do trabalho do coração, freqüentemente comprometido, ao reassumir suas funçōes no periodo pós-operatório imediato. 
REIS. C. L.; ÉVORA, P. R. B.; RIBEIRO, P. J. F.; BRASIL, J. C. F.; OTAVIANO, A. G.; BONGIOVANI, H. L.; BOMBONATO. R.: PEREIRA, A. S. - Contrapulsação aórtica intraoperatória pós circulação extracorpórea (CEC): apresentação de método. Rev. Bras. Cir. Cardiovasc., 1(2):29-33, 1986.

REIS, C. L.; ÉVORA, P. R. B.; RIBEIRO, P. J. F.; BRASIL, J. C. F.; OTAVIANO, A. G.; BONGIOVANI, H. L.; BOMBONATO, R.; FERREIRA, A. S. - Intra-operative, post-extracorporeal aortic counterpulsation: presentation of the method. Rev. Bras. Cir. Cardiovasc., 1 (2):29-33, 1986.

ABSTRACT: The authors present on a system to obtain the aortic counterpulsation-effect in the immediate period after cardiopulmonary bypass for cardiac surgery.

DESCRIPTORS: aortic counterpulsation, intraoperative; aortic counterpulsation; extracorporeal circulation.

\section{REFERÊNCIAS BIBLIOGRÁFICAS}

1 BIRTWELL, W. C.; SOROFF, H. S.; RUIZ, U.; MANY, M.; GIRON, F.; DETERLING Jr., R. A. - Pressión sincrónica auxiliada. In: FRIEDBERG, C. K. Progresos en las enfermedades cardiovasculares. Barcelona, Editorial Científico Medica, 1969, p. 357.

BURMAN, S. O. \& ELIAS, D. O. - Assistência circulatória mecânica no choque. In: SCHUMMER, W. \& NYHUS, L. Tratamento do choque. São Paulo, Editora Manole, 1976. p. 207.

3 ÉVORA, P.R. B.; REIS, C. L.; RIBEIRO, P. J. F.; BONGIOVANI, H. L.; BRASIL, J. C. F.; OTAVIANO, A. G.; PEREIRA, A. S.; FEREZ, M. A. - Fluxo pulsátil em circulaçāo extracorpórea (CEC): apresentaçāo de um sistema mecânico com estudos preliminares hemodinâmicos e de transporte de oxigênio. Arq. Bras. Cardiol, 43 (Supl. 1): 34, 1984. (Resumo)

4 REIS, C. L.; ÉVORA, P. R. B.; RIBEIRO, P. J. F.; BRASIL, J. C. F.; OTAVIANO, A. G.; BONGIOVANI, H. L.; MARGARIDO, E. A. - Fluxo pulsátil em circulação extracorpórea: apresentação de um sistema mecânico com estudos preliminares hemodinâmicos e do transporte de oxigênio. Arq. Bras. Cardiol., 43 (4): 239-244. 1984.

5 REIS, C. L.; ÉVORA, P. R. B.; RIBEIRO, P. J. F.; BRASIL, J. C. F.; OTAVIANO, A. G.; SILVA, J, R. P.; MARGARIDO, E. A.; BONGIOVANI, H. L., BOMBONATO, R. - Apresentação de um sistema mecânico para obten. çâo de fluxo pulsátil em circulação extracorpórea: nota prévia. Rev. Soc. Cardiol. (Ribeirăo Preto), 5: (1, 2 , 3 e 4): 22-25, 1981.

6 REIS, C. L; ÉVORA, P. R. B.; RIBEIRO, P. J. F.; OTAVIANO, A. G.; BONGIOVANI, H. L.; BOMBONATO, R. - A mechanical system for pulsatile cardiopulmonary bypass. J. Cardiovasc. Surg. (Torino) (aceito para publicação).

7 SKINNER, J. R.; ZEFF, R. H.; KONGTAHWORN, C.; TOON, R. S.; SMITH, C. D.; PHILLIPS, S. J. - A technique for immediate manual external aortic counterpulsation during cardiac surgery. Ann. Thorac. Surg., 38 (3): 296-297, 1984.

\section{Discussão}

DR. KENJI NAKIRI

Sāo Paulo, SP.

Cumprimentamos o Dr. Celso Luis dos Reis e seus colaboradores, por esta interessante contribuição à cirurgia cardíaca. As vantagens da circulação assistida por contrapulsação já são por demais conhecidas de todos. Entretanto, ainda nos dias de hoje, persiste controvérsia quanto à superioridade do fluxo pulsátil sobre o não-pulsátil. Ao rever a literatura, tenho ficado com a impressão de que os parâmetros utilizados na avaliação comparativa desses dois tipos de fluxos não são totalmente adequados. Ao meu ver, os estudos seriam mais precisos se quantificassem fenômenos que ocorrem em nível arteriolar e capilar.

(Slide) $\mathrm{Em}$ um trabalho realizado juntamente com o Dr. De Paeppe, há alguns anos, no Instituto do Coração, observamos arteríolas de córtex cerebral de cães submetidos a fluxo não-pulsátil e pulsátil. Constatamos que, com fluxo não-pulsátil, ocorreu uma diminuição de $40-50 \%$ do diâmetro arteriolar, com desaparecimento da pulsatividade, evidentemente. Com fluxo pulsátil, o comportamento foi diferente. Imprimindo-se um tempo sistólico de $30 \%$ à pulsatilidade, não houve alteração no diâmetro arteriolar, além de se observarem variações sistodiastólicas do seu diâmetro. Porém, quando se alterou o tempo sistólico, por exemplo, para $70 \%$, houve alteração de aproximadamente $40 \%$ do diâmetro arteriolar, com perda da pulsatilidade.

(Slide) Este slide mostra que, quando a arteríola perde a pulsatilidade, o fluxo cai acentuadamente. À medida que se acentuam as variações sistodiastólicas do diâmetro arteriolar, o fluxo aumenta. Portanto, quando se fala em fluxo pulsátil, é preciso referir-se às suas características. 
REIS, C. L.; ÉVORA, P. R. B.; RIBEIRO, P. J. F.; BRASIL, J. C. F.; OTAVIANO, A. G.; BONGIOVANI, H. L.; BOMBONATO, R.; PEREIRA, A. S. - Contrapulsação aórtica intraoperatória pós circulação extracorpórea (CEC): apresentação de método. Rev. Bras. Cir. Cardiovasc., 1(2):29-33, 1986.

(Slide) Para imprimir fluxo pulsátil à circulação extracorpórea, nós construímos um balão de poliuretano contido em um estojo rígido, um sistema semeIhante ao do Dr. Reis.

(Slide) Aqui, se mostra a aplicação do sistema em uma cirurgia, em uma criança. A propulsão sincronizada é realizada por um controle eletropneumático, também desenvolvido no Instituto do Coração. A contrapulsação proposta pelo Dr. Reis consiste, na realidade, em imprimir maiores amplitudes nas variaçōes sistodiastókicas da pulsatilidade, amortecidas pelo baixo débito. Desejaria, então, fazer duas perguntas: 1) Por quais parâmetros ele se guia para estabelecer um modo de contrapulsação "ideal" em seu sistema? 2) A cânula aórtica, especialmente em crianças, constitui fator limitante para um sistema deste tipo. Tem observado alguma dificuldade? Agradeço à Comissão Organizadora o privilégio de tecer estes comentários.

DR. REIS

(Encerrando)

Nossos agradecimentos aos comentários do Prof. Kenji Nakiri. É motivo de grande orgulho para nossa Instituição tê-lo como comentador oficial do nosso trabalho, neste Congresso, valorizando-o, uma vez que o Prof. Kenji é sabidamente uma auto- ridade no campo experimental de assuntos congêneres ao aqui apresentado. Concordamos com o Dr. Kenji, quanto aos aspectos controversos das reais vantagens do fluxo pulsátil em circulação extracorpórea. Em trabalho anterior (Arq. Bras. Cardiol., 43:238, 1984), não encontramos vantagens quanto aos parâmetros de perfusão capilar periférica propostos por Shoemaker, em perfusões até 60 minutos, embora tenhamos encontrado evidências da diminuição da resistência vascular periférica, nas perfusões em que se utilisou o fluxo pulsátil por tempo superior a um hora. Quanto ao sistema de fluxo pulsátil empregado pelo Prof. Kenji, já o conhecíamos e, fundamentalmente, ele apresenta duas diferenças, em relação ao nosso mecanismo: 1) o nosso bulbo pulsátil é de borracha atóxica e não de poliuretano; 2) o sincronismo da pulsaçāo é inteiramente mecânico, acoplado à manivela do rolete arterial da bomba de CEC. Gostariamos de ressaltar que o trabalho apresentado não é sobre fluxo pulsátil em CEC, e sim uma adaptação do dispositivo por nós desenvolvido, para auxílio mecânico temporário ao ventrículo esquerdo por contrapulsação intra-operatória. Neste aspecto, o parâmetro utilizado para estabelecer-se a contrapulsação foi o clássico, ou seja, o perfil de pulso obtido por cateterização da artéria radial, a partir da qual se posicionou a onda de contrapulsação. Em relação ao emprego do sistema em crianças, ainda não temos experiência que permita conclusões. 